\title{
Preface to the GRG special issue on quantum gravity
}

\author{
Hermann Nicolai • George F. R. Ellis
}

Published online: 11 March 2009

(C) The Author(s) 2009. This article is published with open access at Springerlink.com

The present volume collects contributions from the 405th WE-Heraeus-Seminar "Quantum Gravity: challenges and perspectives" which took place in Bad Honnef, Germany 14-16 April 2008. The purpose of this meeting was to bring together leading experts from different approaches to quantum gravity, and to discuss these approaches in an open and constructive spirit. We would like to specially thank all speakers who contributed a survey article to the present volume, but also those who finally could not make it, as well as the session chairs and all participants for the lively discussions. We believe that the articles collected here not only reflect the spirit of the meeting, but also provide up-to-date and pedagogical introductions to the various topics, thus giving a very balanced overview of the current attempts to come to grips with the main challenge of 21 st century theoretical physics. Thanks are also due to the WE-Heraeus foundation for generous support of the meeting.

It was during this meeting that we learnt of the death of J.A. Wheeler, one of the true pioneers of quantum gravity. As we had anyhow planned to devote a special issue of GeRG to this subject, it was spontaneously decided to dedicate it to the memory of J.A. Wheeler. For this reason we also decided to include a short obituary by C.A. Misner together with an interview that had been conducted with Wheeler by J. Bicak and was originally published in Czech. We hope that readers will enjoy this special issue, and that it is a fitting tribute to the towering achievements of J.A. Wheeler.

Open Access This article is distributed under the terms of the Creative Commons Attribution Noncommercial License which permits any noncommercial use, distribution, and reproduction in any medium, provided the original author(s) and source are credited.

\footnotetext{
H. Nicolai $(\bowtie)$

MPI for Gravitational Physics, Golm, Germany

e-mail: Hermann.Nicolai@aei.mpg.de

G. F. R. Ellis

University of Cape Town, Rondebosch, South Africa

e-mail: George.Ellis@uct.ac.za
} 